\title{
NON-VANISHING OF CARLITZ-FERMAT QUOTIENTS MODULO PRIMES
}

\author{
NGUYEN NGOC DONG QUAN
}

1. Introduction. Let $q=p^{s}$, where $p$ is a prime and $s$ is a positive integer. Let $\mathbb{F}_{q}$ be the finite field of $q$ elements, and set $A=\mathbb{F}_{q}[T]$ and $k=\mathbb{F}_{q}(T)$. Let $\tau$ be the mapping defined by $\tau(x)=x^{q}$, and let $k\langle\tau\rangle$ denote the twisted polynomial ring. Let $C: A \rightarrow k\langle\tau\rangle\left(a \mapsto C_{a}\right)$ be the Carlitz module, namely, let $C$ be an $\mathbb{F}_{q}$-algebra homomorphism such that $C_{T}=T+\tau$. Let $R$ be any commutative $k$-algebra. The definition of the Carlitz module $C$ implies that $C_{T}(a)=T a+a^{q}$ for every $a \in R$.

Let $\wp$ be a monic prime in $A$. The Carlitz-Fermat quotient $\mathcal{Q}_{\wp}$ : $A \rightarrow A$ is the mapping defined by

$$
\mathcal{Q}_{\wp}(a):=\frac{C_{\wp-1}(a)}{\wp} \quad \text { for each } a \in A .
$$

The notion of Carlitz-Fermat quotients first appeared in the work of Mauduit [6]. In this note, we prove several non-vanishing results of Carlitz-Fermat quotients modulo primes in $A$, which are Carlitz module analogues of the results in [5]. As a by-product, we give an alternative proof of the result in [2] that a Mersenne prime in $A$ is a non-Wieferich prime in the Carlitz module context. We briefly recall the notions of Mersenne primes and Wieferich primes in the Carlitz module setting.

Definition 1.1. A Mersenne prime $M$ in $A$ is a prime of the form $\alpha C_{\wp}(1)$, where $\wp$ is a monic prime in $A$ and $\alpha$ is an element in $\mathbb{F}_{q}^{\times}$.

Definition 1.2. Let $W$ be a prime element in $A$. Write $W=\alpha \wp$, where $\alpha \in \mathbb{F}_{q}^{\times}$is the leading coefficient of $W$ and $\wp$ is a monic prime in $A$. We say that $W$ is a Wieferich prime if $\mathcal{Q}_{\wp}(1) \equiv 0(\bmod \wp)$; otherwise, $W$ is called a non-Wieferich prime.

2010 AMS Mathematics subject classification. Primary 11G09, 11R58, 11 T55.

Keywords and phrases. Carlitz module, Carlitz-Fermat quotients, Mersenne prime, Wieferich prime.

Received by the editors on May 9, 2014. 
The notion of Mersenne primes in $A$ was introduced by the author in [2], and the notion of Wieferich primes in $A$ was first introduced by Dinesh Thakur in [9]. See also Thakur's recent preprint [11] for more beautiful results on several types of primes in $A$ and their connections with zeta values.

2. Carlitz-Fermat quotients. In this section, we prove several properties of Carlitz-Fermat quotients. The main result of this section is the following.

Proposition 2.1. Let $\wp$ be a monic prime in $A$ of degree $d>0$. Then

(i) $\mathcal{Q}_{\wp}$ is an $\mathbb{F}_{q}$-module homomorphism;

(ii) $\mathcal{Q}_{\wp}(a+m \wp) \equiv \mathcal{Q}_{\wp}(a)-m(\bmod \wp)$ for all $a, m \in A$; and

(iii) $\mathcal{Q}_{\wp}\left(C_{m}(a)\right) \equiv m \mathcal{Q}_{\wp}(a)(\bmod \wp)$ for all $a, m \in A$.

Proof. Since the Carlitz module $C$ is an $\mathbb{F}_{q}$-algebra homomorphism, we see that (i) follows immediately.

We now prove (ii). By [8, Proposition 12.11], one can write $C_{\wp}(x) \in A[x]$ in the form

$$
C_{\wp}(x)=\wp x+[\wp, 1] x^{q}+\cdots+[\wp, d-1] x^{q^{d-1}}+x^{q^{d}},
$$

where $[\wp, i]$ is a polynomial of degree $q^{i}(d-i)$ for each $1 \leq i \leq d-1$. Furthermore, we know that $[\wp, i]$ is divisible by $\wp$ for each $1 \leq i \leq d-1$. Hence, we see that

$$
\begin{aligned}
C_{\wp-1}(m \wp) & =\wp(m \wp)+[\wp, 1](m \wp)^{q}+\cdots+(m \wp)^{q^{d}}-m \wp \\
& =\wp\left(m \wp+[\wp, 1] m^{q} \wp^{q-1}+\cdots+m^{q^{d}} \wp^{q^{d}-1}-m\right),
\end{aligned}
$$

and thus $\mathcal{Q}_{\wp}(m \wp) \equiv-m(\bmod \wp)$. It thus follows from part (i) that

$$
\mathcal{Q}_{\wp}(a+m \wp)=\mathcal{Q}_{\wp}(a)+\mathcal{Q}_{\wp}(m \wp) \equiv \mathcal{Q}_{\wp}(a)-m \quad(\bmod \wp) .
$$

We now prove that (iii) holds. Let $m$ be an arbitrary element in $A$ of degree $h$, and let $a \in A$. We can write $C_{m}(x) \in A[x]$ in the form $C_{m}(x)=m x+[m, 1] x^{q}+[m, 2] x^{q^{2}}+\cdots+[m, h-1] x^{q^{h-1}}+[m, h] x^{q^{h}}$, 
where $[m, i]$ is a polynomial of degree $q^{i}(h-i)$ for each $1 \leq i \leq h$. We see that

$$
\begin{aligned}
\wp \mathcal{Q}_{\wp}\left(C_{m}(a)\right) & =C_{\wp-1}\left(C_{m}(a)\right)=C_{m(\wp-1)}(a) \\
& =C_{m}\left(C_{\wp-1}(a)\right)=C_{m}\left(\wp \mathcal{Q}_{\wp}(a)\right) \\
& =m\left(\wp \mathcal{Q}_{\wp}(a)\right)+[m, 1]\left(\wp \mathcal{Q}_{\wp}(a)\right)^{q}+\cdots+[m, h]\left(\wp \mathcal{Q}_{\wp}(a)\right)^{q^{h}},
\end{aligned}
$$

and thus

$$
\mathcal{Q}_{\wp}\left(C_{m}(a)\right)=m \mathcal{Q}_{\wp}(a)+[m, 1] \wp^{q-1}\left(\mathcal{Q}_{\wp}(a)\right)^{q}+\cdots+[m, h] \wp^{q^{h}-1}\left(\mathcal{Q}_{\wp}(a)\right)^{q^{h}} .
$$

Therefore, we deduce that

$$
\mathcal{Q}_{\wp}\left(C_{m}(a)\right) \equiv m \mathcal{Q}_{\wp}(a) \quad(\bmod \wp),
$$

which proves that (iii) is true.

Remark 2.2. Let $p$ be an odd prime in $\mathbb{Z}$. Recall that the Fermat quotient $q_{p}: \mathbb{Z} \rightarrow \mathbb{Z}$ is defined by $q_{p}(a)=\left(a^{p-1}-1\right) / p$ for each integer $a$ with $\operatorname{gcd}(a, p)=1$. According to [1], Eisenstein noted that the Fermat quotient $q_{p}$ satisfies the following properties.

(1) $q_{p}(a b) \equiv q_{p}(a)+q_{p}(b)(\bmod p)$;

(2) $q_{p}(a+m p) \equiv q_{p}(a)-m / a(\bmod p)$;

and

(3) $q_{p}\left(a^{m}\right) \equiv m q_{p}(a)(\bmod p)$.

There are well-known analogies $[\mathbf{3}, \mathbf{8 ,} \mathbf{1 0}]$ between the Carlitz module $a \mapsto C_{m}(a), m \in A$, and the power map $a \mapsto a^{m}, m \in \mathbb{Z}$. Hence, (i), (ii) and (iii) in Proposition 2.1 are Carlitz module analogues of (1), (2) and (3) mentioned above.

\section{Non-vanishing of Carlitz-Fermat quotients modulo primes.} In this section, using Proposition 2.1, we prove several non-vanishing results of Carlitz-Fermat quotients modulo primes.

Theorem 3.1. Let $\wp$ be a monic prime in $A$ of degree $d>0$, and let $\mathcal{Q}_{\wp}$ be the Carlitz-Fermat quotient of $\wp$. Let $a, m$ be nonzero elements in $A$ such that $\wp$ does not divide $m$. Assume that $C_{m}(a)=b \wp$ for some 
$b \in A$. Then

$$
\mathcal{Q}_{\wp}(a) \equiv-\frac{b}{m} \quad(\bmod \wp)
$$

Proof. It follows from part Proposition 2.1 (iii) that

$$
m \mathcal{Q}_{\wp}(a) \equiv \mathcal{Q}_{\wp}\left(C_{m}(a)\right)=\mathcal{Q}_{\wp}(b \wp) \quad(\bmod \wp)
$$

By parts (i) and (ii) in Proposition 2.1, we deduce that $\mathcal{Q}_{\wp}(b \wp) \equiv-b$ $(\bmod \wp)$, and thus $\mathcal{Q}_{\wp}(a) \equiv-b / m(\bmod \wp)$.

In [2], the author proves that a Mersenne prime is a non-Wieferich prime in the Carlitz module context. We present here an alternative proof of this result using Theorem 3.1.

Corollary 3.2. Let $M_{P}=\alpha C_{P}(1)$ be a Mersenne prime, where $\alpha$ is an element in $\mathbb{F}_{q}^{\times}$and $P$ is a monic prime in $A$ of degree $d>0$. Then $M_{P}$ is a non-Wieferich prime.

Proof. Write $M_{P}=\beta \wp$, where $\beta \in \mathbb{F}_{q}^{\times}$is the leading coefficient of $M_{P}$ and $\wp$ is a monic prime in $A$. We see that $C_{P}(1)=\alpha^{-1} M_{P}=$ $\alpha^{-1} \beta \wp$. We can write $C_{P}(x) \in A[x]$ in the form

$$
C_{P}(x)=P x+[P, 1] x^{q}+\cdots+[P, d-1] x^{q^{d-1}}+x^{q^{d}},
$$

where $[P, i]$ is a polynomial in $A$ of degree $q^{i}(d-i)$ for each $1 \leq i \leq d-1$. Furthermore, it is known [4, Proposition 2.4] that $[P, i]$ is divisible by $P$ for each $1 \leq i \leq d-1$. Hence, we deduce that

$$
\begin{aligned}
\beta \wp & =M_{P}=\alpha C_{P}(1) \\
& =\alpha(P+[P, 1]+\cdots[P, d-1]+1) \equiv \alpha \quad(\bmod P),
\end{aligned}
$$

and thus $\wp \equiv \alpha \beta^{-1} \not \equiv 0(\bmod P)$.

Since $P, \wp$ are relatively prime, applying Theorem 3.1 with $P, \wp, 1$ and $\alpha^{-1} \beta$ in the roles of $m, \wp, a$ and $b$, respectively, we deduce that

$$
Q_{\wp}(1) \equiv-\frac{\alpha^{-1} \beta}{P} \not \equiv 0 \quad(\bmod \wp),
$$

and thus $M_{P}=\beta \wp$ is a non-Wieferich prime.

Corollary 3.3. Let $a$ be an element in $A$, and let $m, n$ be nonzero elements in $A$. Let $H$ be the unique element in $A$ such that $C_{m n}(a)=$ 
$C_{n}(a) H$. Assume that there exists a monic prime $\wp$ dividing $H$ such that $\wp$ does not divide $m n$. Write $H=b \wp$ for some $b \in A$. Then

$$
\mathcal{Q}_{\wp}(a) \equiv-\frac{b C_{n}(a)}{m n} \quad(\bmod \wp) .
$$

Proof. We see that $C_{m n}(a)=H C_{n}(a)=b C_{n}(a) \wp$. Since $\wp$ does not divide $m n$, applying Theorem 3.1 with $a, b C_{n}(a), m n$ and $\wp$ in the roles of $a, b, m$ and $\wp$, respectively, we deduce that

$$
\mathcal{Q}_{\wp}(a) \equiv-\frac{b C_{n}(a)}{m n} \quad(\bmod \wp) .
$$

Corollary 3.4. We maintain the same notation and assumptions as in Corollary 3.3. Assume that $v_{\wp}(H)=1$, where $v_{\wp}$ denotes the $\wp$ adic valuation. Assume further that $m$ and $C_{n}(a)$ are relatively prime. Then $\mathcal{Q}_{\wp}(a) \not \equiv 0(\bmod \wp)$.

Proof. By Corollary 3.3, we know that

$$
\mathcal{Q}_{\wp}(a) \equiv-\frac{b C_{n}(a)}{m n} \quad(\bmod \wp) .
$$

We prove that $b C_{n}(a) \not \equiv 0(\bmod \wp)$. Indeed, we know that $1=$ $v_{\wp}(H)=v_{\wp}(b \wp)=1+v_{\wp}(b)$, and thus $v_{\wp}(b)=0$. Hence, $b \neq \equiv$ $(\bmod \wp)$.

We can write $C_{m}(x) \in A[x]$ in the form

$$
C_{m}(x)=m x+[m, 1] x^{q}+\cdots+[m, \operatorname{deg}(m)] x^{q^{\operatorname{deg}(m)}},
$$

where $[m, i]$ is a polynomial of degree $q^{i}(\operatorname{deg}(m)-i)$ for each $1 \leq i \leq$ $\operatorname{deg}(m)-1$ and $[m, \operatorname{deg}(m)]$ is the leading coefficient of $m$. Then we see that

$$
\begin{aligned}
C_{m n}(a) & =C_{m}\left(C_{n}(a)\right) \\
& =C_{n}(a)\left(m+[m, 1]\left(C_{n}(a)\right)^{q-1}+\cdots+[m, \operatorname{deg}(m)]\left(C_{n}(a)\right)^{q^{\operatorname{deg}(m)}-1}\right) .
\end{aligned}
$$

Since $C_{m n}(a)=C_{n}(a) H$, we deduce that

$$
H=m+[m, 1]\left(C_{n}(a)\right)^{q-1}+\cdots+[m, \operatorname{deg}(m)]\left(C_{n}(a)\right)^{q^{\operatorname{deg}(m)}-1} .
$$

Since $\operatorname{gcd}\left(m, C_{n}(a)\right)=1$, it follows from the equation of $H$ that $H \equiv m \not \equiv 0(\bmod \mathfrak{q})$ for each prime $\mathfrak{q}$ dividing $C_{n}(a)$. Hence, $H$ and 
$C_{n}(a)$ are relatively prime, and therefore $C_{n}(a) \not \equiv 0(\bmod \wp)$. This implies that $b C_{n}(a) \not \equiv 0(\bmod \wp)$, and hence

$$
\mathcal{Q}_{\wp}(a) \equiv-\frac{b C_{n}(a)}{m n} \not \equiv 0 \quad(\bmod \wp) .
$$

Remark 3.5. Corollary 3.3 and Corollary 3.4 are Carlitz analogues of Corollary 2 and Corollary 3 in [5], respectively.

\section{REFERENCES}

1. L.E. Dickson, History of the theory of numbers, Volume I: Divisibility and primality, Chelsea Publishing Company, New York, 1966.

2. N.N. Dong Quan, Carlitz module analogues of Mersenne primes, Wieferich primes, and certain prime elements in cyclotomic function fields, J. Num. Theor. 145 (2014), 181-193.

3. D. Goss, Basic structures of function field arithmetic, Ergeb. Math. Grenzg. 35, Springer-Verlag, Berlin, 1996.

4. D.R. Hayes, Explicit class field theory for rational function fields, Trans. Amer. Math. Soc. 189 (1974), 77-91.

5. W. Johnson, On the nonvanishing of Fermat quotients $(\bmod p)$, J. reine angew. Math. 292 (1977), 196-200.

6. V. Mauduit, Quotients de Fermat-Carlitz, C.R. Acad. Sci. Paris 321 (1995), $1139-1141$.

7. , Carmichael-Carlitz polynomials and Fermat-Carlitz quotients, in Finite fields and applications, Lond. Math. Soc. Lect. Note 233, Cambridge University Press, Cambridge, 1996.

8. M. Rosen, Number theory in function fields, Grad. Texts Math. 210, SpringerVerlag, New York, 2002.

9. D.S. Thakur, Iwasawa theory and cyclotomic function fields, in Arithmetic geometry Contemp. Math. 174, American Mathematical Society, Philadelphia, 1994.

10. , Function field arithmetic, World Scientific Publishing Co., Inc., River Edge, NJ, 2004.

11. Fermat versus Wilson congruences, arithmetic derivatives and zeta values, preprint, 2014.

Department of Mathematics, University of Texas at Austin, Austin, TX 78712

Email address: dongquan.ngoc.nguyen@gmail.com 